\title{
Osteomyelitis of the Mandible in a Patient with Osteopetrosis. Case Report and Review of the Literature
}

\author{
Carlos Moreno García • María Asunción Pons García • \\ Raúl González García · Florencio Monje Gil
}

Received: 6 December 2009/ Accepted: 3 March 2011/Published online: 20 April 2011

(C) Association of Oral and Maxillofacial Surgeons of India 2011

\begin{abstract}
Osteopetrosis is a rare hereditary bone disorder presenting with variable clinical features and is characterized by an increase in bone density and reduction of marrow spaces that result from a defect in the function of osteoclasts and, consequently, a decrease in bone turnover. This disease is generally divided into three types: severe infantile malignant autosomal recessive, intermediate mild autosomal recessive, and benign autosomal dominant. The prognosis of the first two types is very poor and is characterized by an early onset, usually within the first decade of life, and early death. The benign-type is characterized by a later onset and a longer life span. Ten percent of osteopetrosis cases develop osteomyelitis that usually involves the mandible. The osteomyelitis is generally caused by tooth extraction or pulpal necrosis. The leading cause of the increased rate of infection is thought to be a lack of adequate bone vasculature. Treatment of osteomyelitis secondary to osteopetrosis is controversial. Treatment regimens include high-dose systemic antibiotics coupled with thorough debridement of necrotic bone and primary closure of soft tissues, if possible. Hyperbaric oxygen has been used for the treatment of chronic osteomyelitis.
\end{abstract}

C. M. García ( $\varangle)$ · R. G. García · F. M. Gil

Department of Oral and Maxillofacial Surgery-Head and Neck

Surgery, University Hospital Infanta Cristina, Badajoz, Spain

e-mail: carlosmorenogar@gmail.com

M. A. P. García

Department of Neurology, University Hospital Infanta Cristina,

Badajoz, Spain

\section{Introduction}

Osteopetrosis, also known as Albers-Schönberg disease, osteopetrosis generalisata [1] and marble bone disease [2, 3] was first described in 1904 by the German radiologist Albers-Schönberg [1, 4-6] as a bone disease with an increase in cortical bone mass at the expense of the medullary bone. In 1926, Karshner [7] introduced the term osteopetrosis to describe this disease [8]. It is a rare hereditary bone disorder [1, 6] presenting with variable clinical features and is characterized by an increase in bone density and reduction of marrow spaces that result from a defect in the function of osteoclasts [1, 4], and consequently, a decrease in bone turnover [6,9]. It has been shown that the normal amount of osteoclasts are present, but that the cells are inactive or incompetent $[8,10]$.

The disease represents a spectrum of clinical variants due to the heterogeneity of genetic defects resulting in osteoclast dysfunction. Classic osteopetrosis occurs in two varieties described as malignant and benign diseases [11]. Malignant osteopetrosis is transmitted as a mendelianrecessive trait and is diagnosed at birth or during early childhood. They had a generalized increase in bone density and myelofibrosis suffering from hepatosplenomegaly, anemia, thrombocytopenia, and neurologic manifestations like optic atrophy [12]. They usually die during the first years of age because of anemia or secondary infection [13]. At the moment, allogenic hemopoietic stem cell transplantation is the only curative treatment of autosomal recessive osteopetrosis and should be offered as early as possible [12, 14].

The benign variety of osteopetrosis is transmitted as a mendelian-dominant trait, develops later, and is diagnosed at third or fourth decade of life by means of a routine roentgenograms. There are two forms differentiated by 
clinical and radiological signs. Autosomal dominant osteopetrosis (ADO) type I is characterized by a pronounced and symmetrical osteosclerosis of the skull and an enlarged thickness of the cranial vault [15]. Clinically, ADO type I is the only type of osteopetrosis not associated with increased fracture rate $[16,17]$. Less sclerosis of the skull was found in type II (Albers-Schöberg disease) and it was more pronounced in the base. Clinical manifestations of ADO type II are dominated by long-bone fractures, which occur, with or without trauma, in $78 \%$ of the patients [18]. Other classic manifestations of ADO type II include hip osteoarthritis, facial nerve palsy, and mandibular osteomyelitis $[2,12,18]$.

An intermediate type described by Beighton et al. [19] is more prevalent in practice. Tips and Lynch [20] reported no racial or sexual predisposition. Malfunction of osteoclastic activity results in excessive formation of immature bone, thickening of the cortical bones, and narrowing or obliteration of the medullary cavities. It is believed that osteoclasts fail to release the necessary lysosomal enzymes for bone resorption into the extracellular space [21, 22]. Defects in different genes have been described that lead to a phenotype with osteopetrosis. These defects include mutations in the gene encoding carbonic anhydrase II, the proton pump gene and the chloride channel gene. Recently, the immune response has been incriminated in the pathogenesis of various metabolic bone diseases, including osteopetrosis.

Both cytotoxic $\mathrm{T}$ lymphocyte-associated antigen 4 and programmed death-1, a newly identified immunoregulatory receptor, have been shown to negatively regulate immune responses, and to affect osteoclastogenesis and bone remodelling $[23,24]$. The clinical presentation and radiological picture may vary according to the severity of the disease.

\section{Case Report}

A 26 years old white male was referred to the Department of Oral and Maxillofacial Surgery, University Hospital Infanta Cristina of Badajoz, Spain for evaluation and treatment of chronic abscess in the right side of the face. The patient explained that the process started 6 months ago after the extraction a molar of the mandible. Facial examination revealed an infection of the mandible and a wide abscess in the submandibular region of the right side of the face with draining fistula. Intraoral examination revealed extensively exposed necrotic bone with sequestrum in the right mandibular molar area. Past medical history revealed the diagnosis of ADO, with numerous bone fractures. The patient was bearer of a hip joint prosthesis (Figs. 1, 2).

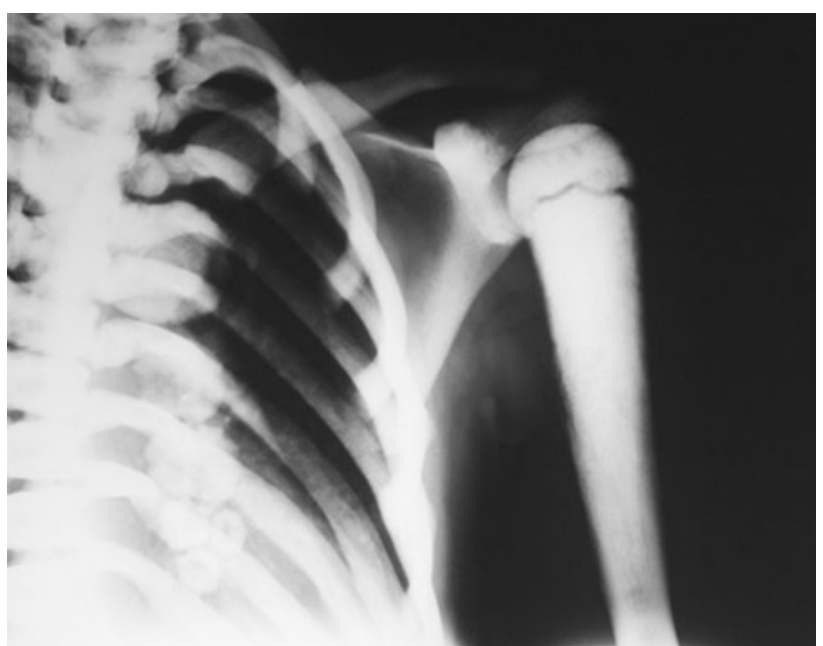

Fig. 1 Past medical history revealed numerous bone fractures

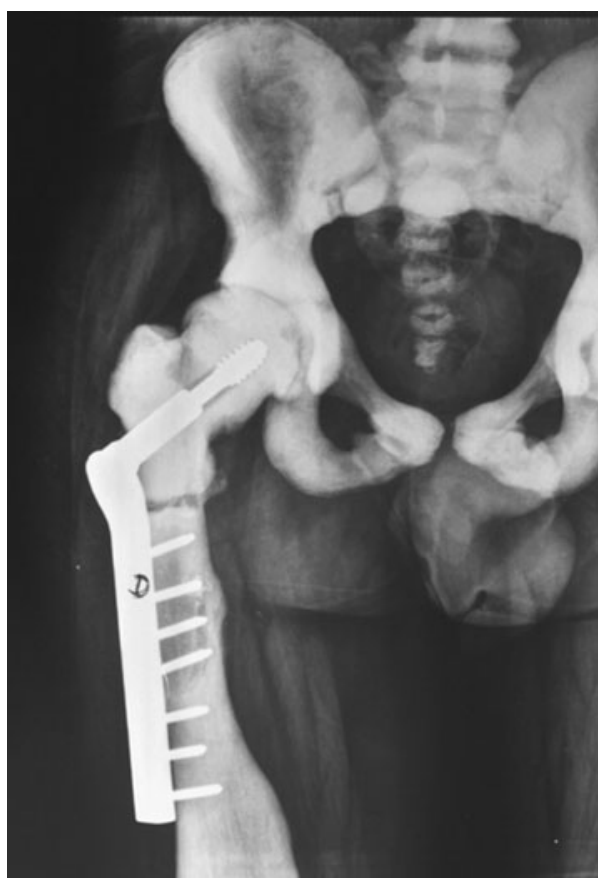

Fig. 2 The patient was bearer of a hip joint prosthesis

A radiolucent, poorly demarcated image demonstrating evidence of sequestrum in the posterior right side of the mandible suggestive of a chronic osteomyelitis was observed in the panoramic radiograph (Fig. 3). An increase in bone density, with obliteration of the medullary spaces and loss of distinction between the cortex and medulla was present in the CT-scan. A hypodense image was observed in the posterior region of the right side of the mandible, showing destruction of the vestibular cortical suggestive of chronic osteomyelitis (Fig. 4).

A biopsy was performed. The histopathology was reported as consistent with osteomyelitis. The treatment 


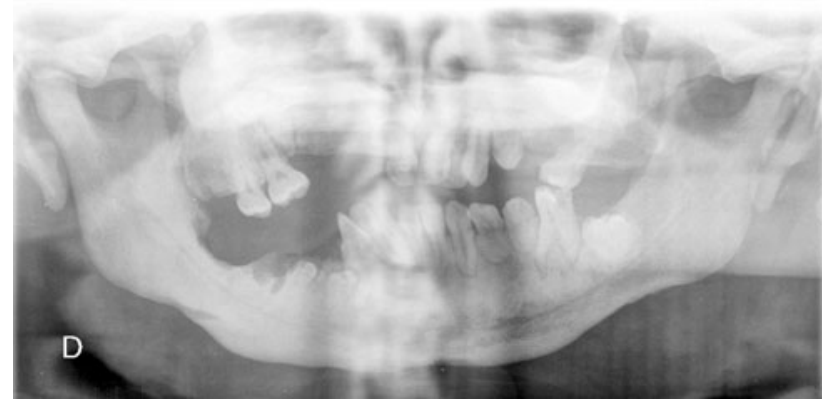

Fig. 3 Panoramic radiograph showing sclerosis of the jaws, great distortion in permanent teeth, hypercementosis and evidence of bone sequestrum on the posterior right side of the mandible

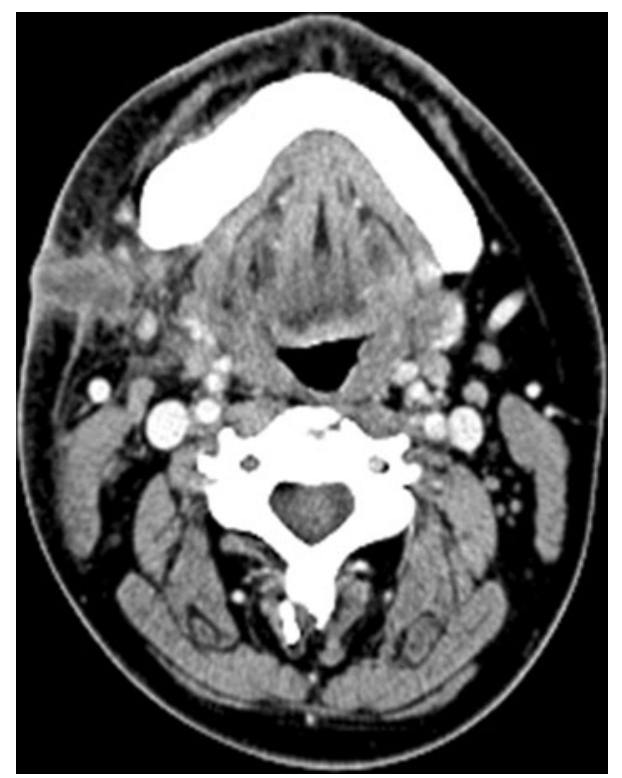

Fig. 4 CT showed a generalized increase in bone density and in the posterior region of the right side of the mandible was seen a hypodense image suggestive of abscess

consisting in intravenous antibiotic therapy (clindamicyn), debridement of the necrotic bone and sequestrum and drainage of the abscess. He was submitted to a systemic antibiotic regimen and daily irrigation of the osteomyelitis region with iodine. Subsequently the patient maintained the same antibiotic by oral administration for 10 days.

The chronic osteomyelitis remained unresolved for the past 3 months and acute episodes are managed with antibiotic therapy (Co-amoxiclav, Metronidazole...). Hyperbaric oxygen was used, with poor results. The condition has maintained a chronic course for the last 12 months.

\section{Discussion}

The osteopetrosis are caused by a diminished activity of osteoclasts, which results in defective remodelling of bone and increased bone density [12]. Osteopetrosis is generally divided into three types: severe infantile malignant autosomal recessive, intermediate mild autosomal recessive, and benign autosomal dominant $[3,25]$. The prognosis of the first two types is very poor and is characterized by an early onset, usually within the first decade of life, and early death. The benign-type is characterized by a later onset and a longer life span $[3,6]$.

Dominant forms are more common [26]. Two subtypes of ADO are reported based on radiographical features [27]. In type I (ADO I), there are generalized, diffuse osteosclerosis affecting mainly the cranial vault [27], due to mutation in the gene located in chromosome 11q12-13, precisely in the region where a high-bone-mass syndrome has been localised [28]. Type II (ADO II), the form originally described in 1904 by Albers-Schönberg [29], is the most common form with an estimated prevalence of up to $5.5 / 100,000$. A gene residing in chromosome $16 \mathrm{p} 13.3$ encoding the ClCN7 chloride channel [11], essential for the acidification of the extracellular resorption lacuna of osteoclast [30], is mainly defective in ADO II [31].

There are three clinically distinct forms of autosomal recessive osteopetrosis (ARO) [32]. The most common ARO (also called the malignant type) [33], has severe manifestations, and presents in the infantile age group, presumably due to mutations either in the TCIRG1 gene which encode for the $\alpha 3$ subunit of the vacuolar $\mathrm{H}(+)$ ATPase or in the ClCN7 gene encoding an osteoclastspecific chloride channel [34]. These patients have bone marrow compromise as a result of bone overgrowth in the marrow space. They usually die from anemia with congestive heart failure, or sepsis in their infancy or childhood. The increased susceptibility to severe infection is presumably related to pancytopenia secondary to marrow space obliteration. The second ARO type with carbonic anhydrase II deficiency is associated with renal tubular acidosis and cerebral calcification, extramedullary haematopoiesis, hepatosplenomegaly and pancytopenia. The third recessive type is milder, presenting in childhood with variable orthopaedic and dental symptoms. They tend to have radiographical evidence of the disease, short stature, macrocephaly, increased upper segment/lower segment ratio, decreased arm span, mandibular prognathism, nerve compression, and a tendency for developing fractures and osteomyelitis. Of the 18 affected family members in 11 families with this form reported so far, many children were asymptomatic with only radiographical disease. In many of these cases, there was parental consanguinity [31, 32].

In osteopetrosis, the determining factor for healing is the vascularity of the bone. Consequently, in such patients the healing process is slow, the outcome often unfavorable, and the bone becomes susceptible to infection [35]. Osteomyelitis is a well-recognized complication of 
osteopetrosis [2, 3, 36, 37, 38, 39], and the general dental practitioner should be aware that the reduced vascularity of bone and impaired white cell function might lead to the development of osteomyelitis in patients with osteopetrosis. The most common site of involvement is the mandible, and it is associated with dental extractions or surgical exposure of the pathologic bone $[1,6]$.

Patients with osteopetrosis frequently visit the dentists for several complications like dental caries, delayed eruption and early loss of teeth, enamel hypoplasia, malformed roots and crowns, and thickening of the lamina dura; with the most common problem being caries. Constriction of the canals housing neurovascular bundles supplying the teeth and jaws as well as obliteration of the marrow cavities and dental pulp chambers, lead to bone necrosis and dental caries [31], and ultimately develop osteomyelitis in $10 \%$ of cases. Osteomyelitis may be potentially severe with a protracted course due to the accompanying anemia and neutropenia [31].

The change in bone structure is accompanied by a marked tendency toward fragility, and fractures may be sustained even in trivial accidents [35]. The pathologic fractures in patients with osteopetrosis are likely the result of structural weakness associated with poorly organized bone and persistent accumulations of immature bone and calcified cartilage. With progression of the disease, the bones become increasingly sclerotic and opaque on radiographs, the latter feature giving rise to the term "marble bone" $[4,35]$. The frontal and nasal bones may be dense and somewhat enlarged, and the air sinuses may be obscured [1, 35]. According to Bakeman et al. [3], osteopetrosis radiographically appears as an increased density of the entire skeleton (e.g., ribs, pelvis, clavicle, femur, skull base, and jaws). The long bones show increased cortical thickening and decreased marrow space, and become clubshaped. The pelvis and the scapula may show endobone formations [3]. The presence of hypercementosis, involving different teeth, was also mentioned, was reported in the literature only by Smith $[6,35]$.

The malignant form presents with devastating symptoms early in childhood and rapid worsening of the condition, resulting in a short lifespan, whereas the benign form may be diagnosed late in childhood with a variety of prominent clinical features, such as frontal bossing, leonine facies, malocclusion of teeth and hepatosplenomegaly [39]. Atypical features may include microcephaly and a normal upper segment to lower segment ratio. A radiological skeletal survey usually reveals increased bone density with poor differentiation between the cortex and the medulla. The defective remodelling process characteristic of osteopetrosis has many clinical implications in the head and face regions. Cranial imaging of autosomal recessive osteopetrosis shows small optic canals, orbits and nasoethmoid complex. The paranasal sinuses are either poorly pneumatized, like the mastoid air cells, or show bud formation [40] Areas of condylar cartilage calcification may be seen [1]. These radiological characteristics of underdevelopment may support the theory that delayed maturation is the primary morphological abnormality in osteopetrosis and that bone thickening is a secondary manifestation to reduced bone turnover. Of importance in the head and neck region is the stenosis and compression of the cranial foramina, resulting in multiple cranial palsies. The optic, olfactory, trigeminal, facial and cochlear nerves are most commonly affected. Cummings et al. [41] reported the case of a sixmonth-old infant who was diagnosed with malignant autosomal recessive osteopetrosis and was found to have optic nerve pallor secondary to orbital fissure narrowing (seen on computed tomography of the brain) [24].

Batra and Shah [42] reported a case of osteomyelitis of the mandible following tooth extraction in a 19-year-old woman with malignant osteopetrosis. This result was attributed to the poor bone vascularization and reduced local defences. Most teeth were unerupted and the paranasal sinuses were not aerated (as seen on computed tomographic evaluation of the facial bones). The initial clinical diagnosis was a draining gingivobuccal fistula; however, failure to respond to broad-spectrum antibiotics and the pathological description of the debrided bony fragments of the right maxilla shifted the diagnosis to chronic osteomyelitis. The chronicity of this infection has necessitated repeated surgical intervention for further debridement of the necrotic tissues and infected bone [24].

Ten percent of osteopetrosis cases develop osteomyelitis that usually involves the mandible. The osteomyelitis is generally caused by tooth extraction or pulpal necrosis. The leading cause of the increased rate of infection is thought to be a lack of adequate bone vasculature. Treatment of the infection is difficult because of the large amounts of poorly vascularized bone with gradual obliteration of the marrow space in the affected regions. Multiple draining fistulae and bony sequestrum are common clinical finding [8]. Osteomyelitis of the maxilla is very rare, probably because of the thin cortical bone and rich collateral blood supply [12].

Radiographically, the typical brugger jersey spineQ and endobones (bbone within a boneQ) were seen in the pelvis of the patients with ADO type II. These alterations are not present in ADO type I patients. On the other hand, radiographs in patients with ADO type I showed a pronounced sclerosis of the skull with an enlarged thickness of the cranial and facial walls positively correlated to age. The sclerosis of the skull in type II was most striking at the base. Laboratory findings in ADO usually show the characteristic of a myelophthisic anemia due to the obliteration of hemopoietic marrow cavities [2, 5, 24, 43]. 
Important differential diagnoses considered are pyknodysostosis, metaphyseal dysplasia, diaphyseal sclerosis, melorheostosis, osteopetrosis striata, osteopoikilosis, Engelmann's disease and infantile cortical sclerosis [31].

Treatment of osteomyelitis secondary to osteopetrosis is controversial. Treatment regimens include high-dose systemic antibiotics coupled with thorough debridement of necrotic bone and primary closure of soft tissues, if possible [2-4]. Hyperbaric oxygen (HBO) has been used for the treatment of chronic osteomyelitis [3, 4]. Mechanisms of $\mathrm{HBO}$ action in osteomyelitis include enhanced leucocytic killing, osteoclastic resorption of the dead osteomyelitic tissue, fibroblastic division, collagen production, neovascularisation, and enhanced permeation of certain antibiotics (aminoglycosides) across bacterial cell walls within the necrotic tissue. As osteoclasts are 100 times more metabolically active than osteocytes, its function is highly oxygen dependant [31]. There are few reports demonstrating the success of treatment; in many cases, the osteomyelitis remains unresolved indefinitely [2, 12]. In a review of 57 cases of osteomyelitis resulting from osteopetrosis, most cases were found to be chronic and resistant to treatment [2]. Unfortunately, there seems to be no definitive treatment for osteopetrosis of the maxilla or mandible without complete removal of the affected bone. In order to minimize such problems, patients with osteopetrosis should be encouraged to maintain good dental care and oral hygiene, because there is a potential risk of promoting osteomyelitis if surgical procedures are performed [6].

Management of osteopetrosis has to be individualised because of the wide spectrum of clinical symptoms and complications. Medical management of osteopetrosis revolves around modulation of the osteoclasts, either to stimulate the remaining host osteoclasts or to provide an alternative source of the same. Restriction of calcium intake [44], high-dose calcitriol therapy, steroids, parathyroid hormone and recombinant human interferon gamma-1b, have all been attempted to stimulate the host osteoclasts with variable success rate.

Use of a microvascularized osseous free flap may be favorable but may be precluded because of absence of a suitable donor site in these patients [12].

Palliative treatment, including nerve decompression and debridement, seems to be the best course of action. The best treatment appears to be preventive management with routine dental care. Teeth should be endodontically treated, if possible, rather than extracted, because periosteal stripping of bone may predispose asymptomatic bone to become necrotic and to sequester. Any debridement should be as conservative as possible, removing only grossly necrotic bone through limited flap dissections. Because the disease process is systemic, there is often no clear delineation between affected and unaffected bone [9].
Recently, bone marrow transplantation has been successfully used in the treatment of malignant osteopetrosis, offering hope to those so afflicted; [6, 45] it has been curative in a significant percentage of patients but an acceptable donor can be found in only about $40 \%$ [31].

\section{References}

1. Elster AD, Theros EG, Key LL et al (1992) Cranial imaging in autosomal recessive osteopetrosis. Part I. Facial bones and calvarium. Radiology 183:129-135

2. Barry CP, Ryan CD (2003) Osteomyelitis of the maxilla secondary to osteopetrosis: report of a case. Oral Surg Oral Med Oral Pathol Oral Radiol Endod 95:12-15

3. Bakeman RJ, Abdelsayed RA, Sutley SH et al (1998) Osteopetrosis:a review of the literature and report of a case complicated by osteomyelitis of the mandible. J Oral Maxillofac Surg 56:1209-1213

4. Juggins KJ, Walton GM, Patel M (2001) Osteomyelitis complicating osteopetrosis e a case report. Dent Update 28:509-511

5. Steiner M, Gould AR, Means WR (1983) Osteomyelitis of mandible associated with osteopetrosis. J Oral Maxillofac Surg 41:395-405

6. Portela MA, Santana E, Jorge WA, Paraiso M (2006) Osteomyelitis of the mandible associated with autosomal dominant osteopetrosis: a case report. Oral Surg Oral Med Oral Pathol Oral Radiol Endod 102:94-98

7. Karshner RG (1926) Osteopetrosis. AJR 16:405-419

8. Long RG, Ziccardi V (2001) Osteopetrosis of the maxilla. Oral Surg Oral Med Oral Pathol Oral Radiol Endod 91:139-140

9. Battaglia MA, Drigo P, Laverda AM et al (1991) Osteomielite e osteopetrosis infantile. Descrizione di un caso. Minerva Stomatol 40:125-127

10. Shapiro F, Glimcher MJ, Holtrop ME, Tashjian AH, BrickleyParsons D, Kenzora JE (1980) Human osteopetrosis: a histological, ultrastructural, and biochemical study. J Bone Joint Surg 62:384-399

11. Benichou OD, Cleiren E, Gram J et al (2001) Mapping of autosomal dominant osteopetrosis Type II (Albers-Schfnberg disease) to chromosome 16p13.3. Am J Hum Genet 69:647-654

12. Junquera L, Rodríguez-Recio C, Villarreal P, García-Consuegra L (2005) Autosomal dominant osteopetrosis and maxillomandibular osteomielitis. Am J Otolaryngol Head Neck Med Surg 26:275-278

13. Stocks RM, Wang WC, Thompson JW et al (1998) Malignant infantile osteopetrosis. Arch Otolaryngol Head Neck Surg 124:684-689

14. Driessen GJ, Gerritsen EJ, Fischer A et al (2003) Long-term outcome of haematopoietic stem cell transplantation in autosomal recessive osteopetrosis: an EBMT report. Bone Marrow Transplant 32:657-663

15. Bollerslev J, Grontved A, Andersen PE Jr (1988) Autosomal dominant osteopetrosis: an otoneurological investigation of the two radiological types. Laryngoscope 98:411-413

16. Bollerslev J, Andersen PE Jr (1988) Radiological, biochemical and hereditary evidence of two types of autosomal dominant osteopetrosis. Bone 9:7-13

17. Van Hul E, Gram J, Bollerslev J et al (2002) Localization of the gene causing autosomal dominant osteopetrosis type I to chromosome 11q12-13. J Bone Miner Res 17:1111-1117

18. Benichou OD, Laredo JD, de Vernejoul MC (2000) Type II autosomal dominant osteopetrosis (Albers-Schfnberg disease): 
clinical and radiological manifestations in 42 patients. Bone 26:87-93

19. Beighton P, Horan F, Hamersma H (1977) A review of the osteopetroses. Postgrad Med J 53:507-516

20. Tips RL, Lynch HT (1962) Malignant congenital osteopetrosis resulting from a consanguineous marriage. Acta Paediatr 51:585-588

21. Marks SC Jr (1973) Pathogenesis of osteopetrosis in the rat: reduced bone resorption due to reduced osteoclast function. Am J Anat 138:165-189

22. Schofield BH, Levin LS, Doty SB (1974) Ultrastructure and lysosomal histochemistry of ia rat osteoclasts. Calcif Tissue Res 14:153-160

23. Nagahama K, Aoki K, Nonaka K et al (2004) The deficiency of immunoregulatory receptor PD-1 causes mild osteopetrosis. Bone 35:1059-1068

24. Hamdan A, Nabulsi M, Farhat F, Haidar R, Fuleihan N (2006) When bone becomes marble: head and neck manifestations of osteopetrosis. Paediatr Child Health 11(1):37-40

25. Shaff MI, Mathis JM (1982) Osteomyelitis of the mandible. an initial feature in late-onset osteopetrosis. Arch Otolaryngol 108:120-121

26. Bollerslev J (1989) Autosomal dominant osteopetrosis: bone metabolism and epidemiological, clinical, and hormonal aspects. Endocr Rev 10:45-67

27. Bollerslev J, Andersen PE Jr (1988) Radiological, biochemical and hereditary evidence of two types of autosomal dominant osteopetrosis. Bone 91:7-13

28. Van Hul E, Mathysen D, Bollerslev J, Gram J, Van Hul W (2000) Autosomal dominant osteopetrosis type I is genetically linked to the same region on human chromosome 11 as the high bone mass phenotype. J Bone Miner Res 15(suppl 11):S260

29. Albers-Schönberg HE (1904) Röntgenbilder einer seltenen Knockenerkrankung. Munch Med Wochenschr 51:365-368 German

30. Kornak U, Kasper D, Bosl MR et al (2001) Loss of the ClC-7 chloride channel leads to osteopetrosis in mice and man. Cell 104:205-215

31. Chattopadhyay P, Kundu AK, Saha AK, Karthak RO (2008) Mandibular osteomyelitis and multiple skeletal complications in Albers-Schönberg disease. Singapore Med J 49(9):e229-e233
32. Kahler SG, Burns JA, Aylsworth AS (1984) A mild autosomal recessive form of osteopetrosis. Am J Med Genet 17:461-464

33. Gerritsen EJ, Vossen JM, van Loo IH et al (1994) Autosomal recessive osteopetrosis: variability of findings at diagnosis and during the natural course. Pediatrics 93:247-253

34. Sly WS, Hewett-Emmett D, Whyte MP, Yu YSL, Tashian RE (1983) Carbonic anhydrase II deficiency identified as the primary defect in the autosomal recessive syndrome of osteopetrosis with renal tubular acidosis and cerebral calcification. Proc Natl Acad Sci USA 80:2752-2756

35. Smith NH (1966) Albers-Schönberg disease (osteopetrosis). Oral Surg Oral Med Oral Pathol 22:699-710

36. Gupta DS, Gupta MK, Borle RM (1986) Osteomyelitis of the mandible in marble bone disease. Int J Oral Maxillofac Surg 15:201-205

37. Lawoyin DO, Daramola JO, Ajagbe HA et al (1988) Osteomyelitis of the mandible associated with osteopetrosis: report a case. Br J Oral Maxillofac Surg 26:330-335

38. Hinds EC (1970) Non-inflammatory bone disease. J Oral Surg 28:27-38

39. Shah AM, Boby KF, Karande SC, Lahiri KR, Jain MK (1996) Three sibs with mild variety of osteopetrosis. J Postgrad Med 42:123-125

40. Bartynski WS, Barnes PD, Wallman JK (1989) Cranial CT of autosomal recessive osteopetrosis. AJNR Am J Neuroradiol 10:543-550

41. Cummings TJ, Proia AD (2004) Optic nerve compression in infantile malignant autosomal recessive osteopetrosis. J Pediatr Ophthalmol Strabismus 41:241-244

42. Batra P, Shah N (2004) Recalcitrant osteomyelitis following tooth extraction in a case of malignant osteopetrosis. Int Dent $\mathrm{J}$ $54: 418-423$

43. Brockstedt H, Bollerslev J, Melsen F, Mosekilde L (1996) Cortical bone remodeling in autosomal dominant osteopetrosis: a study of two different phenotypes. Bone 18:67-72

44. Miyamoto RT, House WF (1980) Neurologic manifestations of the osteopetrosis. Arch Otolaryngol 106:210-214

45. Benecke JE (1993) Facial nerve dysfunction in osteopetrosis. Laryngoscope 103:494-497 